\title{
Diversidad de las algas y los macroinvertebrados y su relación con la productividad trófica de la ciénaga Grande de Beté, Medio Atrato, Chocó
}

\section{Diversity of algae and macroinvertebrates and their trophic relation with productivity of the Ciénaga Grande de Beté, Medio Atrato, Chocó}

\author{
Yairton Cuesta Londoño ${ }^{1}$, Arelys Hurtado ${ }^{1}$, Giovanny Ramírez Moreno²

\section{RESUMEN} \\ La Grande de Beté se concibe, como flujos energéticos representados en \\ interacciones de organismos acuáticos que habitan diferentes estratos de la \\ columna de agua. Pese a la importancia que tienen las algas como productores \\ de primer orden y los macroinvertebrados como descomponedores y transfor- \\ madores de materia orgánica, existen vacíos de información alrededor del \\ conocimiento etológico, ecológico y productivo. Con esta contribución, se \\ pretende aportar al conocimiento de la dinámica trófica de la Grande, tomando \\ como base los indicadores ecológicos, la diversidad y composición de las algas \\ y macroinvertebrados, y su relación con la calidad del agua para la vida \\ silvestre, usando los indices de Margalef, Shonnon, Simpson y BMWP. La \\ comunidad de algas de la zona limnética y el espejo de agua de la Grande, \\ estuvo representada por cuatro divisiones y 31 especies, siendo la Chlorophyta \\ la de mayor abundancia con 11 especies. Los macroinvertebrados acuáticos \\ 1. Biólogos Contratistas, Instituto de In- \\ vestigaciones Ambientales del Pacífico \\ (IIAP), Quibdó, Colombia. \\ e-mail: arelyshm@yahoo.es \\ yaculon@yahoo.com \\ 2. Investigador Principal Componente \\ Ecosistémico, Instituto de Investi- \\ gaciones Ambientales del Pacífico \\ (IIAP), Quibdó, Colombia. \\ e-mail: gramirez@iiap.org.co \\ Grupo de Investigación en conocimien- \\ to, manejo y conservación de los \\ ecosistemas del Chocó biogeográfico. \\ Recibido: 14 de junio de 2011 \\ Aceptado: 20 de octubre de 2011
} presentes en las macrófitas se representaron por nueve órdenes, 15 familias y 17 géneros, para un total de 381 individuos, donde el orden más representativo en número de individuos fue el Glossiphoniformes con 131 individuos. A nivel trófico las algas y los macroinvertebrados juegan un papel importante en la producción primaria de la ciénaga Grande de Beté, que se ve soportado por las altas abundancias y la gran diversidad, que permite a este ecosistema tener una oferta de recursos permanentes, y define el establecimiento de una gran variedad de vida, clave para el mantenimiento y subsistencia de peces, aves y demás grupos que ocurren allí.

Palabras clave: Ecosistemas cenagosos; La Grande de Beté; Macroinvertebrados; Algas; Diversidad; Río Atrato; Chocó.

\begin{abstract}
The Grande de Beté conceived as energy flows represented in interactions of aquatic organisms that inhabit different strata of the water column. Despite the importance of algae as producers of first-and macroinvertebrates as decomposers and transformers of organic matter, there are information gaps of knowledge about behavioral, ecological and productive. With this contribution, we intend to carry the knowledge of the trophic dynamics of the Grande, based on ecological indicators, diversity and composition of algae and macroinvertebrates and their relationship to water quality for wildlife, using indices Margalef, Shannon, Simpson and BMWP. The algal community limnological area and water surface of the Grande, was represented by 4 divisions and 31 species, with the most abundant Chlorophyta 11 species. Aquatic macroinvertebrates present in the macrophytes were represented by 9 orders, 15 families and 17 genera, for
\end{abstract}




\section{Bioetnia Volumen 8 No 2 (julio-diciembre), 2011}

a total of 381 individuals, where the order more representative number of individuals was the Glossiphoniformes with 131 individuals. A trophic level algae and macroinvertebrates play an important role in primary production of the Ciénaga Grande de Beté, which is supported by the high abundance and diversity, allowing the ecosystem to have a permanent supply of resources, defining the establishment of a variety of key to maintaining life and livelihoods of fish, birds and other groups that occur there.

Keywords: Wetland ecosystem; La Grande Beté; Macroinvertebrates; Algae; Diversity; Rio Atrato; Chocó.

\section{INTRODUCCIÓN}

Las planicies inundables de los bosques higrofíticos del medio y bajo río Atrato albergan uno de los ecosistemas más importantes a nivel ecológico y productivo. Estos ecosistemas son modelados por un sinnúmero de depresiones, que combinadas con un sistema de alimentación freático eficiente y la mayor expresión de la precipitación del planeta, mantienen el flujo hídrico que irriga las arterias de los complejos cenagosos. En estos, se depositan por arrastre gran parte de la materia orgánica que se incorpora al río Atrato, confiriéndole a las ciénagas el papel de filtros naturales y de espacios altamente oligotróficos, dicho papel hace que al interior de estos particulares ecosistemas se desarrollen diversos organismos con roles ecológicos particulares, de los cuales las algas y los macro invertebrados acuáticos son la base.

Para los complejos cenagosos del Atrato en relación con la composición de la comunidad de macroinvertebrados acuáticos asociados con diferentes estratos del espejo de agua, se han registrado 12 familias como las mejores representadas: Glossiphoniidae, Hydrobiidae, Ancylidae, Planorbidae, Nitidulidae, Cimicidae, Notonectidae, Libellulidae, Philopotamidae, Palaemonidae, Cyprididae, Sphaeromatidae. En relación con las algas microscópicas se informan géneros pertenecientes al plancton y al perifiton asociados con macrófitas $\mathrm{u}$ otros sustratos $\mathrm{y}$ estratos del espejo de agua, y se ha registrado la división Bacillariophyceae con 16 géneros; la división Cyanophyceae con ocho géneros y la división Chlorophyceae con 13 géneros (Cuesta y Cuesta 2001). Registros de CODECHOCO-CORPOURABA (2006). Se identifican para este complejo de humedales 11 géneros que componen el perifiton de las divisiones Bacillariophyta (Diatomeas), Chlorophyta (Algas verdes), Cyanophyta (Algas verde-azules) y Euglenophyta (Euglenas).

Pese a la importancia que tienen las algas como productores de primer orden y los macroinvertebrados como descomponedores, transformadores de materia orgánica y productores de segundo orden, existe un gran vacío de información alrededor de su conocimiento etológico, ecológico y productivo. Es por esto que en esta contribución, se pretende aportar al conocimiento de la dinámica trófica de la ciénaga Grande de Beté, tomando como indicadores ecológicos, la expresión de diversidad y composición de las algas y macroinvertebrados, y la relación existente con la calidad del agua para la vida silvestre.

\section{METODOLOGÍA}

Área de estudio. Los ecosistemas cenagosos se conciben como flujos de energía representados en las interacciones de los organismos que habitan los diferentes estratos de la columna de agua y en las conexiones que presentan con otros sistemas acuáticos. La ciénaga Grande de Beté priorizada para este estudio es una ciénaga principal o de tipo 1, que se conecta directamente a una fuente hídrica principal (río) a través de un caño, de ahí que la composición de sus aguas se encuentre influida por la dinámica del río Atrato y sus afluentes. Se encuentra inmersa en todo un sistema hídrico donde el río es el eje principal y determinante junto con las condiciones climáticas en los cambios de nivel y direcciones de flujo que presenta. Cuenta con un caño bien definido denominado Amé pero a su vez se puede ingresar a ella por el caño Torrobón, que se conecta con otras ciénagas de la zona. El área de estudio para el componente agua abarca desde las zonas riverinas del humedal, pasando por el espejo de agua; estas zonas contienen la información que permite describir muchos aspectos de la dinámica hídrica y biológica del ecosistema en estudio.

Muestreo de algas. Para la colecta de del fitoplancton se realizaron arrastres verticales en la zona limnética de la ciénaga y arrastres horizontales en forma de círculo alrededor de la misma; se utilizoó para esto una red de $90 \mathrm{~cm}$ de longitud y $20 \mathrm{~cm}$ de diámetro, luego se fijó la muestra con una solución de transeau proporción 1:1:1, APHA- AWWAWPCF. 1992; Allan (1995).

Las muestras se identificaron y describieron taxonómicamente hasta el taxón más asequible, se utilizaron las claves taxonómicas especializadas de Rivera et al. (1982), Cox (1996); Krammer y Lange-Bertalot (1986, 1991), LangeBertalot y Genkal (1999), Lange-Bertalot (1993), Krammer (1992), Metzelin y Lange-Bertalot (1998), Zalorca y Maidana (1997). Además, se corroboró la identificación taxonómica de las especies por medio de la utilización de la página de Internet http://www.diatom.acnatsci.org y con ayuda de un microscopio binocular Marca Nikon-YS2-T. Los conteos de las algas microscópicas se realizaron utilizando una célula de recuento tipo Sedgwinck-Rarter, modificada APHA-AWWAWPCF. 1992, cuyos datos se expresaron en número de individuos por mililitros.

Para las algas del perifiton se utilizó el método de remoción y desprendimiento de las algas presentes en las macrófitas, 
aplicando movimientos azarosos en un recipiente y luego se filtró con ayuda de una red tipo plancton (Figura 1); la identificación y conteo se realizó teniendo en cuenta el desarrollo de la técnica empleada para el fitoplancton. Para ambos grupos se tomaron registros fotográficos en laboratorio, con la ayuda de una cámara digital y microscopio óptico.

Muestreos de macroinvertebrados acuáticos. Para su colección se utilizó el método de remoción y desprendimiento de los macroinvertebrados presentes en las macrófitas, con ayuda de movimientos azarosos de las mismas en la columna de agua (Figura 2); para la captura se empleó un tamiz (tools velo suizo) y pinzas entomológicas para la extracción de los macroinvertebrados presentes; para los macroinvertebrados acuáticos presentes en la interface agua-aire, se utilizó una red tipo surber, luego se fijaron en frascos de muestras con una solución de alcohol 70\% con glicerina líquida APHAAWWA-WPCF. 1992; Allan (1995).

Después se realizó la descripción y determinación taxonómica hasta el taxón más asequible teniendo en cuenta que al ser organismos cíclicos, en ocasiones se dificulta alcanzar la categoría taxonómica más baja, para ello se utilizaron las claves taxonómicas Roldan (1988), Edmonson (1959), McCafferty (1981), Pennak(1978), Merrity Cummins (1996), Fernández y Domínguez (2001) y la ayuda de un microestereoscopio Marca MGC-10.
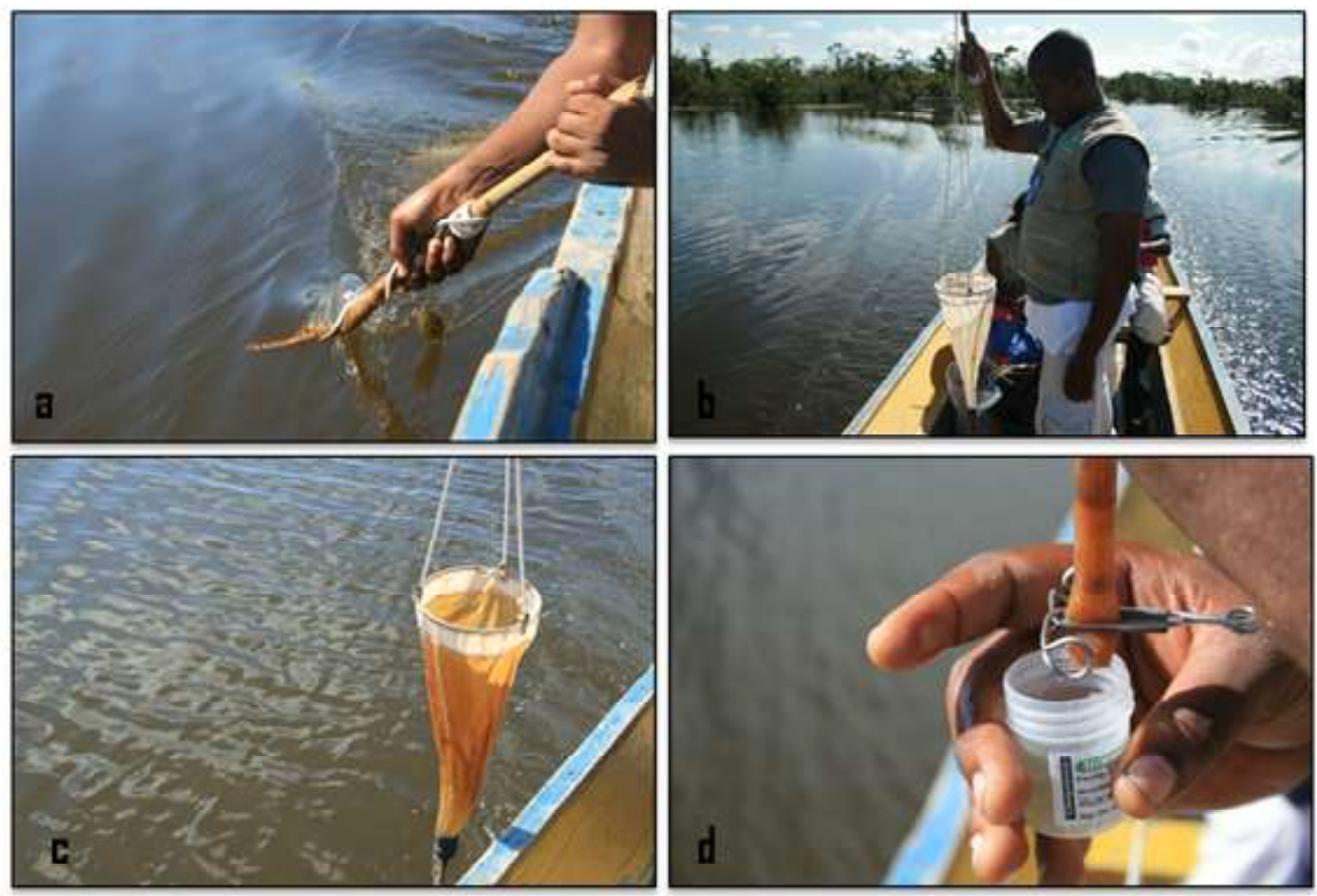

Figura 1. Método de arrastre para muestreo de algas
Es importante anotar, que tanto para macro para algas como para macroinvertebrados se hicieron registros diarios con el fin de determinar el comportamiento de la diversidad en el tiempo.

\section{ANÁLISIS DE DATOS}

Algas y macroinvertebrados acuáticos. Se realizó análisis descriptivo del porcentaje que ocupa cada orden al interior de los muestreos; además, se ejecutó el análisis de índices ecológicos (diversidad de shannom $\mathrm{H}^{\prime}$ y dominancia de Simpson) con el software Past versión 1.15, para explicar la composición de la comunidad, y con la descripción de las familias de macroinvertebrados acuáticos; se analizó la calidad del agua por medio de la aplicación del BMWP (herramienta de trabajo para monitoreo biológico/Col), diseñado para aguas en Colombia (Roldán 1999 y Roldán 2003).

\section{RESULTADOS Y DISCUSIÓN}

Algas del fitoplancton. La comunidad de algas registradas en la zona limnética y en la capa más proximal al espejo de agua de la ciénaga la Grande, estuvo representada por cuatro divisiones y 31 especies, siendo la Chlorophyta la de mayor abundancia con 11 especies, la división Chrysophyta 


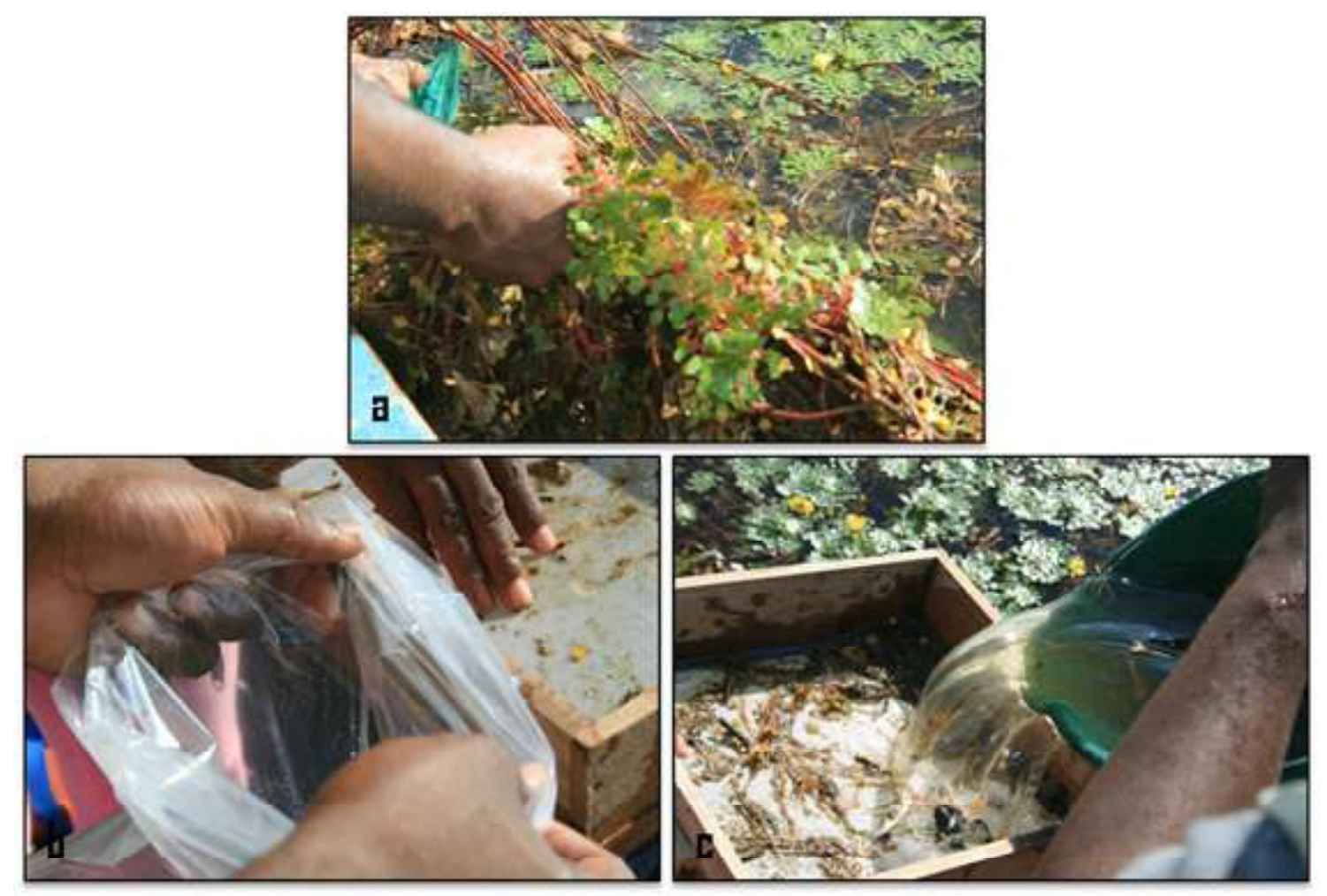

Figura 2. Muestreo de macroinvertebrados

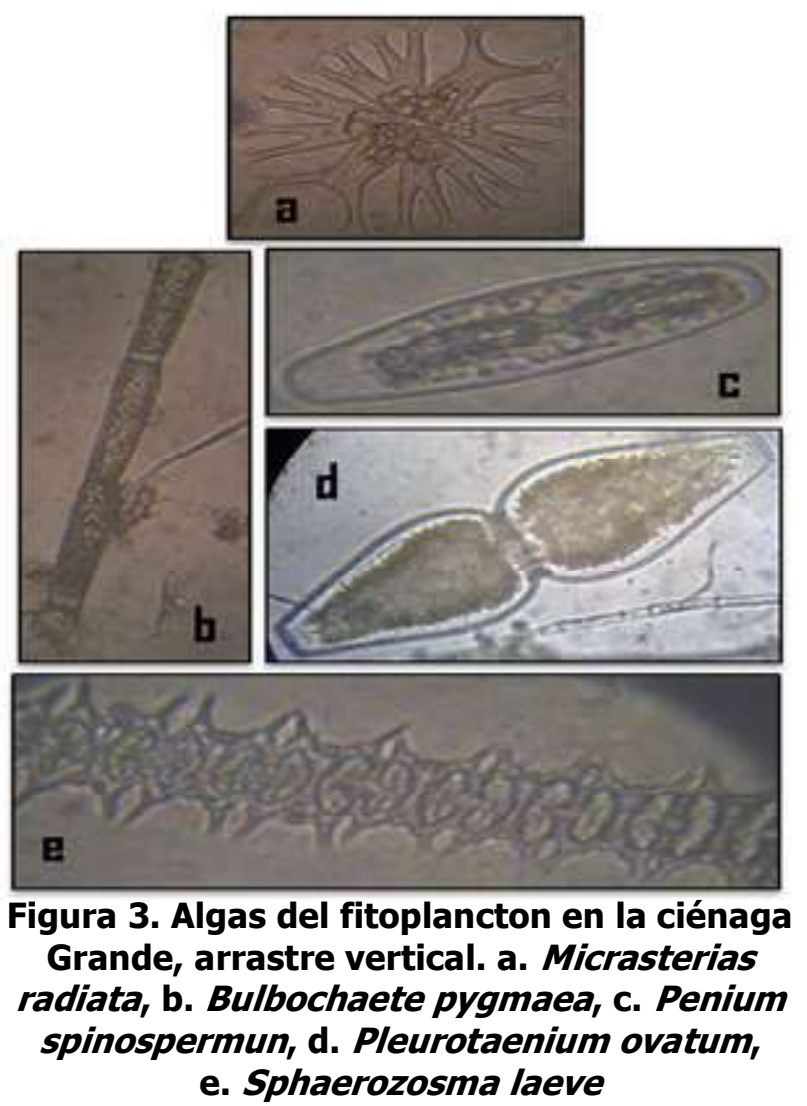

presentó nueve especies, la división Cyanophyta informó siete especies y Euglenophyta registró la abundancia de especie más baja con solo cuatro especies (Figura 3).

La relevancia de la presencia de los grupos de algas microscópicas en el eje vertical de la columna de agua, se describe en relación con la producción de metabolitos secundarios para diferentes organismos en la red trófica, donde la función principal es la absorción de nutrientes inorgánicos, dióxido de carbono y luz solar; es relevante anotar que los niveles de concentración de sólidos disueltos y suspendidos encontrados en la columna de agua no incidieron en la penetración de la radiación solar, fuente principal para el mantenimiento de la gran abundancia de algas, lo que explica una alta producción primaria que se ve reflejada en la complejidad de los niveles tróficos del ecosistema, lo que se corrobora con la descripción de Allan (1995) y Streble y Krauter (1987), donde las tendencias que se observan en las asociaciones de algas del fitoplancton describen la composición trófica del ambiente en relación con la materia orgánica que circula en la ciénaga, la cual suple los requerimientos de nutrientes para que los diferentes grupos de algas se puedan desarrollar, porque algunos tienen requerimientos nutricionales diferentes.

La estructura de la comunidad de algas del fitoplancton en el arrastre vertical, se describe en relación con las tenden- 
Tabla 1

Índices ecológicos para el análisis de la estructura y composición de la comunidad de algas

\begin{tabular}{lrrrrr}
\hline Índices & D 1 & D 2 & D 3 & D 4 & D 5 \\
\hline Taxa & 13 & 13 & 10 & 7 & 16 \\
$\begin{array}{l}\text { Individuos } \\
\text { Índice de }\end{array}$ & 539 & 325 & 396 & 428 & 871 \\
$\begin{array}{l}\text { Shannon } \\
\text { Índice de }\end{array}$ & 2,295 & 2,35 & 2,188 & 1,87 & 2,326 \\
$\begin{array}{l}\text { Simpson } \\
\text { Margalef }\end{array}$ & 0,882 & 0,8869 & 0,8771 & 0,8378 & 0,873 \\
\hline
\end{tabular}

cias que se extraen de los índices ecológicos (Tabla 1), donde la diversidad de Shannon $\left(\mathrm{H}^{1}\right)$, demostró una comunidad con valores relativamente bajos, con referencia a valores medios de diversidad, lo que define una colectividad representada con bajos registros de especies con pocas abundancias, que determinan un estrato pobre en interacciones en la estructura vertical de la ciénaga, reportándose el valor más alto de diversidad $(2,3)$ en los muestreos 1 y 5 , días en los que se registraron algunas especies con abundancias relativamente altas. Un comportamiento similar describió el índice de riqueza de Margalef, en el que el muestreo D5 registró el valor más alto 2,2, describiendo que durante este se registró la mayor variedad de las especies en relación con este índice, con valores que son considerados como propios de zona de baja riqueza.

Las tendencias descritas para los índices anteriores se confirman con los valores obtenidos en el índice de dominancia de Simpson, donde sus estimaciones registran valores relativamente altos para todos los días de estudio, describiendo el dominio colectivo de algunas especies con altas abundancias. Se asume que los valores registrados para los índices ecológicos se deben posiblemente a que los factores que controlan el crecimiento de las algas del fitoplancton en la columna de agua están ejerciendo potencialmente interacciones biológicas, lo que contrasta con lo descrito por Round (1981), quien describe que la incidencia de luz, la herbívora y la disponibilidad de nutrientes afectan la riqueza, abundancia y distribución de las algas.

\section{Macroinvertebrados acuáticos}

Composición y estructura. La comunidad de los macroinvertebrados acuáticos que habitan en las macrófitas de la ciénaga la Grande del municipio del Medio Atrato (Beté), estuvo representada por nueve órdenes, 15 familias y 17 géneros, para un total de 381 individuos, donde el orden más representativo en cuanto al número de individuos fue el

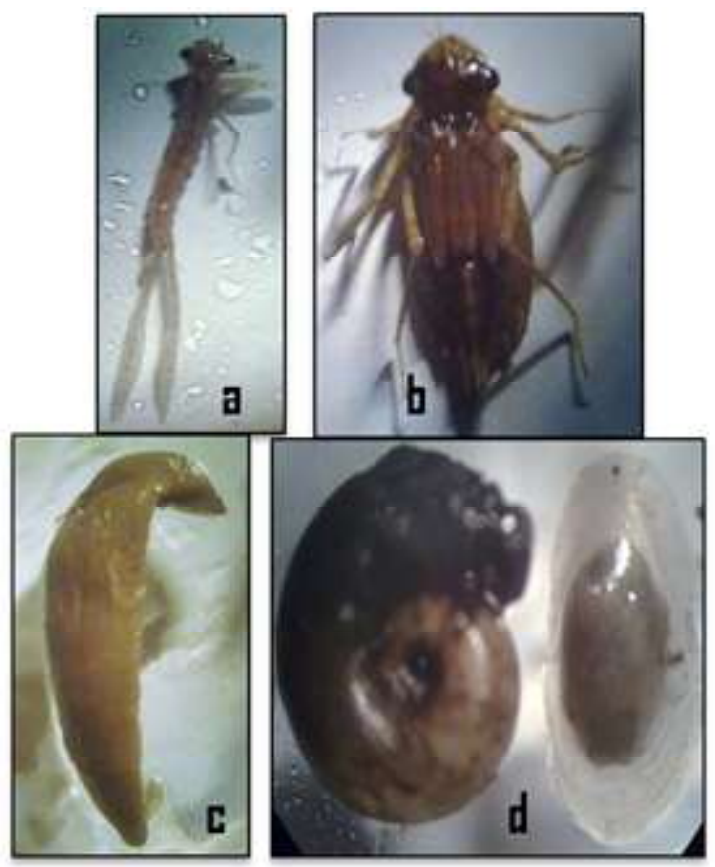

Figura 4. Macroinvertebrados acuáticos a. Acanthagrion sp, b. Brechmoroga sp, c. Hirudinea, d. Hyriidae

Glossiphoniformes con 131, seguido del orden Diptero con 95, Odonata con 57, Hemípteros con 39, Basommatophora con 27, Trichoptera con 14, Unionoida con 12 y el orden Coleoptera con seis individuos (Figura 4).

La composición numérica de la comunidad de macroinvertebrados acuáticos que habitan en las macrófitas presentes en el espejo de agua de la ciénaga la Grande, estuvo representada por los órdenes Odonato que registró tres familias y seis géneros; el orden Trichoptera con dos familias y tres géneros; Díptera con tres familias y dos géneros; Hemíptera dos familias y dos géneros; Coleoptera dos familias y dos géneros y los órdenes Glossiphoniformes, Unionoida y Ephemeroptero con una familia cada uno. La presencia de estos órdenes puede estar relacionada posiblemente con la alta cantidad de materia orgánica presente en la ciénaga, que se configura como fuente de alimento y hábitat, creando vías tróficas alternativas, que dependen del consumo de los macroinvertebrados, esto supone un entorno heterotrófico con presencia de predadores (peces, anfibios y aves) lo que se relaciona con lo expuesto por Wissenger (1999), quien considera que algunos de los patrones complementarios que modelan la estructura y composición de la comunidad de macroinvertebrados en la ciénaga, son la producción primaria, morfología, diversidad y composición vegetal, que ofrecen recursos de alimento, hábitat, reproducción y protección para los macroinvertebrados, contribuyendo así con la abundancia y diversidad de varios organismos acuáticos. 
Bioetnia Volumen 8 No 2 (julio-diciembre), 2011

\section{Tabla 2 \\ Índices ecológicos para el análisis de la estructura y composición de la comunidad}

\begin{tabular}{lrrrrr}
\hline Índices & D 1 & D 2 & D 3 & D 4 & D 5 \\
\hline Taxas & 11 & 15 & 9 & 9 & 7 \\
Individuos & 59 & 137 & 54 & 89 & 54 \\
Índice de & & & & & \\
Shannon & 1,95 & 2,113 & 1,462 & 1,747 & 1,513 \\
Índice de & & & & & \\
Simpson & 0,8055 & 0,8539 & 0,6475 & 0,7527 & 0,7188 \\
\hline
\end{tabular}

Aunque los órdenes registrados en el estudio fueron abundantes en cuanto al número de individuos por género, el orden más representativo en relación con el número de familias y géneros fue el Odonato, lo que expresa disponibilidad de materia orgánica utilizada por estos organismos como rol funcional en la cadena trófica, siendo indicadores de aguas oligotróficas con abundante materia orgánica, lo que concuerda con lo expresado por Roldán (1992), Arango y Roldán (1983), quienes argumentan que el orden Odonato se registra como el más importante en aguas oligomesotróficas, similares a las de la ciénaga estudiada; además son propios de ecosistemas lénticos o de poca corriente, pero son poco resistentes a contaminación, mostrando un panorama apto para el desarrollo de la vida acuática.

La estructura de la comunidad de macroinvertebrados acuáticos, se describe en relación con las tendencias que se extraen de los índices ecológicos (Tabla 2), donde el índice de diversidad de Shannon $\left(\mathrm{H}^{1}\right)$, demostró géneros con valores relativamente bajos, con referencia a valores medios de diversidad, lo que define una colectividad representada con bajos registros de diversidad de géneros, con altas abundancias de algunos géneros durante los días de muestreos. Lo anterior concuerda con la apreciación de Frank (1983) y Richardson (1999), quienes manifiestan que los valores registrados para los índices ecológicos se deben a que la cantidad y la calidad del recurso alimenticio que aporta la vegetación, determina la disponibilidad de nutrientes para la comunidad de macroinvertebrados, por lo que afecta su riqueza y abundancia.

\section{DISTRIBUCIÓN ECOLÓGICA DE LOS MACROINVERTEBRADOS ACUÁTICOS}

La ubicación de los géneros en los diferentes estratos encontrados en las macrófitas, se registra de acuerdo con los recursos disponibles en estas, donde los órdenes Ephemeropteros y Dipteros están asociados con las raíces donde se encuentra gran cantidad de algas y zooplancton que constituyen la dieta básica de este tipo de organismos; los órdenes odonatos, Coleópteros, Trichopteros e Hirudineos, habitan entre los pecíolos y las hojas de las macrófitas, lugar que les permite obtener zooplancton y perifiton para su alimentación, además realizan sus funciones reproductivas y cumplen sus primeros ciclos de vida. Los órdenes Basommatophora y Unionoida se localizan en los tallos de las macrófitas, haciendo Hervivoría en el caso de los primeros y Unionoida aprovecha la frecuencia de peces en este sitio para parasitar en ellos y por último el orden hemíptero se distribuye en la interface agua-aire, donde adquieren recursos alimenticios y se convierte en lugar seguro contra la depredación; vale la pena mencionar que tanto la diversidad como la distribución de estos órdenes permite inferir sobre la oportunidad de recurso y la complejidad trófica que existe para otros grupos que habitan el espejo de agua.

\section{DESCRIPCIÓN DE LA CALIDAD TRÓFICA DE LAS AGUAS DE LA CIÉNAGA LA GRANDE DEL MUNICIPIO DEL MEDIO ATRATO}

La riqueza de macroinvertebrados (15 familias) cumpliendo funciones de descomponedores, predadores y presas, ratifica la importancia ecológica de la ciénaga no solo por la presencia de este grupo, sino también por la gran oferta de hábitat y nichos para cumplir sus funciones ecológicas. La diversidad expresada como riqueza funcional, microambiental y específica, fue la que permitió diagnosticar a través del BMWP, la calidad trófica de las aguas, como muy limpia y de buena calidad ecológica con un valor obtenido de 105 que se encuentra dentro del rango estipulado para dicha calificación, el cual oscila entre 101 y 120 . Es de resaltar que la mayoría de los análisis que se han realizado con la valoración de bioindicación con el BMWP, se ha efectuado en ríos (Alba-Tercedor y Sánchez Ortega 1988; Muñoz y Prat 1996; Prat 1998; Benito de Santos y Puig García 1999), para ecosistemas cenagosos. Todavía existen vacíos de información, es por eso que el presente artículo se convierte en un aporte puntual para el conocimiento de las aguas lénticas de la región.

\section{RECOMENDACIONES PARA SU CONSERVACIÓN}

A nivel trófico las algas y los macroinvertebrados juegan un papel importante en la producción primaria de la ciénaga Grande de Beté, que se ve soportado por las altas abundancias y la gran diversidad, que permiten a este ecosistema tener una oferta de recursos permanentes definiendo el establecimiento de una gran variedad de vida clave para el mantenimiento y subsistencia de peces, aves y demás grupos que ocurren aquí; la especialización de este sistema hace que se 
convierta en el único hábitat de un sinnúmero de macroinvertebrados que ocurren allí de forma permanente y que sin ellos, peces, herpetos y aves no podrían mantener las poblaciones en el buen estado que se observan en la ciénaga Grande de Beté.

Lo anterior refleja la importancia de conservar los tapetes de macrófitas que constituyen el hábitat de algas del perifiton y macroinvertebrados. Al mismo tiempo se hace necesario mantener el sistema aislado de la actividad minera, porque esta actuaría directamente sobre las variables físicoquímicas del agua, espacio donde se desarrollan estos organismos. Asimismo mantener la relación trófica de estos grupos con poblaciones viables de peces y aves permite el control de las abundancias tanto de algas como de macroinvertebrados.

Por último, se hace necesario planificar la investigación en temas como dinámica trófica, producción y biomasa de las algas del plancton, almacenamiento y descomposición de materia orgánica, ciclos biológicos y reproductivos de especies de importancia ecológica y realizar muestreos de algas y macroinvertebrados en otros sustratos (bentónico).

\section{LITERATURA CITADA}

Alba-Tercedor, J. 1996. Macroinvertebrados acuáticos y calidad de las aguas de los rios. IV Simposio del agua en Andalucía (Siaga), Almeria, 1996, Vol. II; p. 203-13.

Allan, J. 1995. Stream ecology. Structure and function running waters. London: Chapman \& Hall; P. 45-107.

Amat, G., E. Blanco. 2003. Artropofauna de los humedales de la sabana de Bogotá. En: Los humedales de Bogotá y la sabana. Volumen 1, Bogotá: Acueducto de Bogotá y Conservación Internacional; p. 91-106.

Apha-Awwa-Wpcf. 1992. Métodos normalizados para el análisis de aguas potables y residuales. Madrid: Diaz De Santos. Ed. 1605 p.

Arango, M.C., G. Roldán, 1983. Odonatos inmaduros del departamento de Antioquia en diferentes pisos altitudinales. Actual Biol. 12 (46): 91-104.

CODECHOCO-CORPOURABA. 2006. Plan de manejo integrado de los humedales del bajo y medio Atrato: municipios de Carmen del Darién, Riosucio, Bojayá y Unguía en el departamento del Chocó y Vigía del Fuerte, Turbo y Murindó en Antioquia. Quibdó: CODECHOCO. 256 p.

Cox, E. 1996. Identification of fresnwater diatoms from live material. Londres: Champan y May. $158 \mathrm{pp}$

Edmonson, W. T. 1959. Free water biology. New York: Edit. Jhon Wiley And Sons; 1247 pp.
Frank, J. H. 1983. Bromeliad Phytoltelmata and their biota, especially mosquitos. H. Frank, P. L. Lounibos (Eds). New Jersey: Plexus Inc. p. 101-3.

Hynes, H. B. N. 1970. The ecology of running waters. Toronto: University of Toronto Press. 555 p.

Kramer, K., H. Lange-Bertalot. 1986. Bacillariphyceae, 1. Teil: Naviculaceae. Veb. Gustav Fisher Verlag, Jena. 576 pp.

Kramer, K., H. Lange-Bertalot. 1991. Bacillariphyceae, 3. Teil: Centrales, Fragilariaceae, Eunotiaceae. Veb. Gustav Fisher Verlag, Jena. 576 pp.

Kramer, K. 1992. Die Gattun Pinnularia In Bayern. Resenburg: Hoppea Bd. 52; $308 \mathrm{pp}$

Lange-Bertalot, H., S. Genkal. 1999. Diatoms from Siberia I. A. R. G. Gantner Verlag K. G. 295 pp.

Lange-Bertalot, H. 1993. 85 Neueu Taxa. Bibliotheca Diatomologica. Band 27

Merritt, R. W., K. W. Cummins. 1996. An introduction to the aquatic insects of North America. Berkeley: Kendall-Hunt Publishing Company. Liwa. Univ. of California; $862 \mathrm{pp}$.

Metzelin, D., H. Lange-Bertalot. 1998. Tropical diatoms of South America Königstein: I. Koeltz Scientific Books; 695 pp.

Mccafferty, W. P. 1981. Aquatic Entomology. Boston: Science Books International; pp. 237-82.

Pennak, R. W. 1978. Freshwater invertebrates of the United States. 2nd. New York: Wiley-Interscience; 803 pp.

Richarson, B. A. 1999. The bromeliad microscom and the assessment of fauna diversity in a neotropical forest. Biotropica. 31: 321-36.

Rivera, P., O. Parra, M. González, V. Dellarrosa, M. Orellana, M. 1982. Manual taxonómico del fitoplancton de las aguas continentales, IV Bacillariophyceae. Chile: Universidad de Concepción; 97 pp.

Roldán, G. 1992. Fundamentos de limnología tropical. Medellín: Editorial Universidad de Antioquia; 403 pp.

Roldán, G. 1996. Guía para el estudio de macro invertebrados en el departamento de Antioquia. Medellín: Edit. Universidad de Antioquia; 217 p.

Roldán, G. 1999. Los macroinvertebrados y su valor como indicadores de calidad de aguas. Ecologia Rev Acad Colomb Cienc. 23 (88): 375-87.

Roldán, G. 2003. Bioindicación de la calidad del agua en Colombia propuesta para el uso del método Bmwp/ Col. Medellin: Editorial Universidad de Antioquia. $170 \mathrm{p}$.

Rivillas, R. J. C., G. Roldán. 2008. Estudio de emergencia de insectos acuáticos en las zonas de bosque ripario, bosque plantado y pastos, Abejorral (Antioquia, Colombia). Rev Acad Colomb Cienc. 32 (122): 105-23.

Round, F. E. 1981. The ecology of algae. Cambridge: Cambridge University Press.

Streble H., D. Krauter. 1987. Atlas de los microorganismos de agua dulce. La vida en una gota de agua. Barcelona: Ediciones Omega, SA.

Wissinger, S. 1999. Ecology of wetland invertebrates. Synthesis and application for conservation and management. En: Batzer, D., R. Rader, S. Wissinger (eds.). Invertebrates in freshwaters wetland of North America ecology and management. New York: John Wiley \& Sons, Inc.

Whitton, B. A. (Ed.) (1975b) River ecology. Berkeley: University of California Press. 725 p.

Zolarca, Y., N. Madaina. 1997. Biblioteca diatomologica, Band 34, Taxonomic and ecological studies of the Paraná River Diatom Flora (Argentina). Berlin-Stuttgart: J. Cramer; 122 pp. 